\title{
Study on Active Vibration Control for High-Speed Elevators*
}

\author{
Naoaki NOGUCHI**, Atsushi ARAKAWA**, Koichi MIYATA***, \\ Takuya YOSHIMURA**** and Seiichi SHIN***** \\ **Mechanical Engineering Research Laboratory, Hitachi,Ltd., \\ 832-2 Horiguchi, Hitachinaka-shi, Ibaraki 312-0034 Japan \\ E-mail: naoaki.noguchi.hu@hitachi.com \\ ***Mito Design \& Production Division, Urban Planning and Development Systems Company, Hitachi,Ltd., \\ 1070 Ichige, Hitachinaka-shi, Ibaraki 312-8506 Japan \\ ****Department of Mechanical Engineering, Tokyo Metropolitan University, \\ 1-1 Minami-osawa, Hachioji-shi, Tokyo 192-0397 Japan

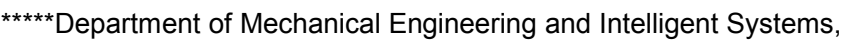 \\ The University of Electro-Communications, \\ 1-5-1 Chofugaoka, Chofu-shi, Tokyo 182-8585 Japan
}

\begin{abstract}
When elevators travel at high speed, horizontal vibrations of the car tend to occur due to guide rail deformations, deteriorating ride comfort. Therefore, several active control systems have been developed to reduce these vibrations. These systems consist of six actuators, which independently move the guide rollers. To reduce costs and installation time, it is necessary to develop a system with a minimum number of actuators and a simplified controller. We developed a system with only three actuators. The controller incorporates an $\mathrm{H}$-infinity control design method for maintaining stability of the system when there is a change in load. Furthermore, from a practical point of view, it is important to reduce the order of the controller so that the time for on-site parameter tuning can be reduced. Therefore, we reduced the $\mathrm{H}$-infinity controller to a $\mathrm{P}$ controller. We demonstrated that the maximum amplitude of vibrations of a car with active control system can be reduced to almost half the vibration amplitude without control.
\end{abstract}

Key words: Elevator, Comfortability in Riding, Vibration Control, Modal Analysis, Optimal Control

\section{Introduction}

High-speed elevators are widely used in high-rise buildings worldwide. In general, vibrations of these elevators due to guide rail deformations tend to be large. To suppress these vibrations, active vibration control systems ${ }^{(1) \sim(4)}$ have been developed. Active control systems with magnetic actuators on each guide roller have disadvantages such as high cost due to the large number of actuators. A further problem is that it takes a long time to tune the many parameters of the controller. To overcome these disadvantages, we determined a new arrangement of actuators and sensors, which effectively suppress vibrations. We propose a system with three actuators and constructed simple models with 7 and 4 degrees of freedom(DOFs) for the controller design. We used an H-infinity control design method to maintain the stability of the system when there is a change in load, and reduced the order of the controller from H-infinity controller to a P controller. We evaluated the performance of our system with numerical simulation and an on-site test run.

Received 29 Sep., 2010 (No. T1-08-1055) Japanese Original : Trans. Jpn. Soc. Mech. Eng., Vol.75, No.754, C (2009), pp.1618-1625 (Received 20 Nov., 2008) [DOI: 10.1299/jsdd.5.164]

Copyright $\odot 2011$ by JSME 


\section{Operational Modal Analysis}

\subsection{Overview of Elevator}

Figure 1 shows a diagram of an elevator car. An elevator car is mainly composed of a cabin and a frame. Isolation rubbers are installed between the cabin and the frame, and roller guide units are installed on the four corners of the frame. Figure 2 shows a diagram of a roller guide unit. It has three rubber rollers; each roller consists of a lever and a spring. The springs absorb vibrations when the car goes over deformed rails.

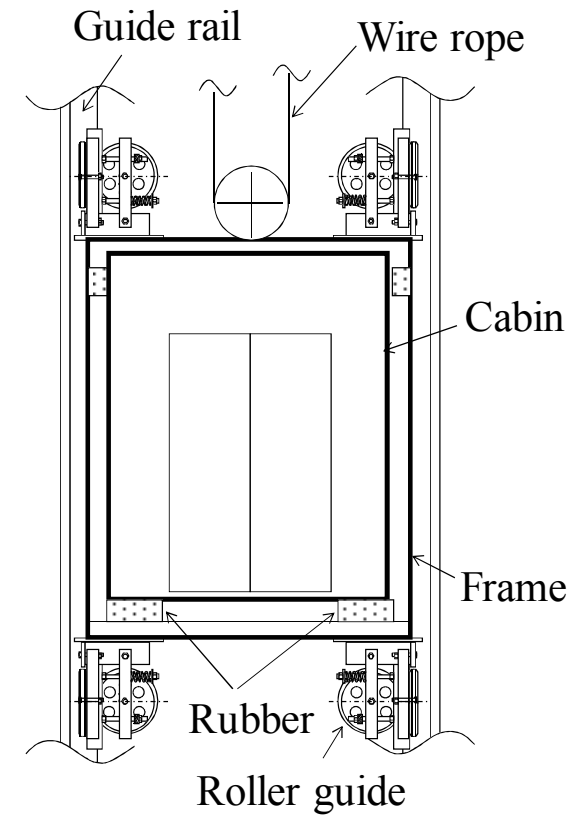

Fig .1 Elevator car
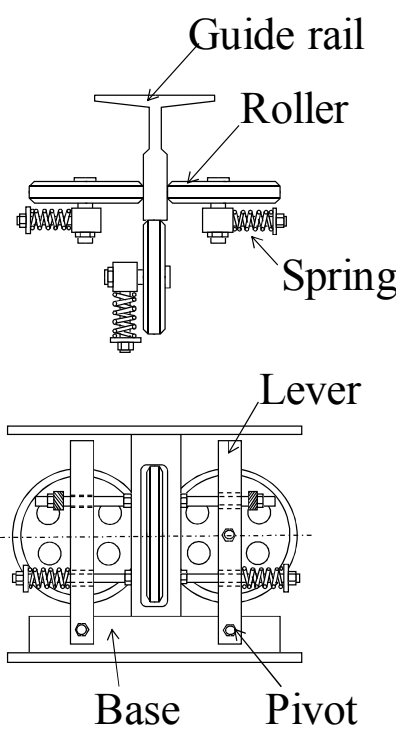

Fig. 2 Roller guide

\subsection{Operational Modal Analysis}

Characteristics, such as natural frequency and modal damping, of structures are generally identified using experimental modal analysis. In this process, frequency response functions (FRFs) are measured by conducting a shaking test. A random input and an impulse input are commonly used as an input signal in the shaking test. However, it is difficult to conduct shaking tests of elevators in construction sites.

To avoid these difficulties, we first apply an operational modal analysis called Frequency Domain Decomposition(FDD) based on the principal component analysis ${ }^{(5)(6)(7)}$. This process uses only vibration response data, involves the estimation of the power spectrum density (PSD) of a single degree of freedom, and specifies the modal characteristics.

We assume vibration data as $\mathbf{X}=\left\{X_{\mathrm{np}}\right\}(\mathrm{n}=1,2, \cdots, \mathrm{N}, \mathrm{p}=1,2, \cdots, \mathrm{P})$, where $\mathrm{N}$ is the number of raw vectors in the data, and $\mathrm{P}$ is the number of elements in each column vector.

The first principal component $z_{1}$ of $\mathrm{N}$-th data as $\mathbf{x}_{\mathbf{n}}=\left(\begin{array}{llll}x_{n 1} & x_{n 2} & \cdots & x_{n P}\end{array}\right)$ is shown as

$$
z_{1}=\sum_{p=1}^{P} \omega_{p 1} x_{n p}=\mathbf{x}_{\mathbf{n}} \boldsymbol{\omega}_{1}
$$

, where $\boldsymbol{\omega}_{1}$ is represented as $\boldsymbol{\omega}_{1}=\left(\begin{array}{llll}\omega_{11} & \omega_{21} & \cdots & \omega_{p 1}\end{array}\right)^{\prime}$.

Applying this relationship to $X=\left\{X_{n p}\right\}$, the first principal component vector $\boldsymbol{\varphi}_{1}$ is

$$
\varphi_{1}=\mathbf{X} \omega_{1}
$$

,where the average and variance of $\boldsymbol{\varphi}_{1}$ are given as follows. 


$$
\begin{aligned}
\tilde{\boldsymbol{\varphi}}_{1} & =\frac{1}{N} \sum_{n=1}^{N} \mathbf{x}_{\mathbf{n}} \boldsymbol{\omega}_{\mathbf{1}} \\
& =\frac{1}{N} \sum_{n=1}^{N}\left(\sum_{p=1}^{P} \omega_{p 1} x_{n p}\right) \\
& =0 \\
\sigma^{2} & =\frac{1}{N-1} \boldsymbol{\varphi}_{\mathbf{1}}{ }^{\mathbf{t}} \boldsymbol{\varphi}_{\mathbf{1}} \\
& =\frac{1}{N-1}\left(\mathbf{X} \boldsymbol{\omega}_{\mathbf{1}}\right)^{T}\left(\mathbf{X} \boldsymbol{\omega}_{\mathbf{1}}\right) \\
& =\boldsymbol{\omega}_{\mathbf{1}}{ }^{\mathbf{t}} \mathbf{V} \boldsymbol{\omega}_{\mathbf{1}}
\end{aligned}
$$

Here, $\mathbf{V}$ is the covariance and described as follows.

$$
\mathbf{V}=\frac{1}{\mathbf{N}-\mathbf{1}} \mathbf{X}^{\mathrm{t}} \mathbf{X}
$$

$\boldsymbol{\varphi}_{1}$ is determined as the variance $\sigma^{2}$ is maximized. Thus, $\boldsymbol{\varphi}_{1}$ is determined by maximizing the following function $J=\varphi_{1}{ }^{\mathrm{t}} \mathbf{V} \boldsymbol{\varphi}_{1}-\lambda\left(\boldsymbol{\varphi}_{1}{ }^{\mathrm{t}} \boldsymbol{\varphi}_{1}-1\right)$. Therefore, eigenvalue analysis which meets the condition $\operatorname{det}|\mathbf{V}-\lambda \mathbf{I}|=0$ is conducted. $\lambda$ and $\boldsymbol{\varphi}_{1}$ represent the eigenvalue and eigen vector, respectively. This gives the following relationship,

$$
\mathbf{V} \boldsymbol{\varphi}_{1}=\lambda \boldsymbol{\varphi}_{1}
$$

By multiplying the conjugate transpose of $\varphi_{1}$, the following equation is obtained.

$$
\varphi_{1}{ }^{\mathrm{H}} \mathbf{V} \varphi_{1}=\lambda \varphi_{1}{ }^{\mathrm{H}} \varphi_{1}=\lambda
$$

Therefore, the variance of $\varphi_{1}$ is given as follows by Eq.(4).

$$
\begin{aligned}
\sigma^{2} & =\varphi_{1}{ }^{\mathrm{t}} \mathbf{V} \boldsymbol{\varphi}_{1} \\
& =\varphi_{1}{ }^{\mathrm{t}} \lambda \boldsymbol{\varphi}_{1} \\
& =\lambda
\end{aligned}
$$

By considering this relationship in the frequency domain, variance $\sigma^{2}$ is equivalent to the power spectrum density of the frequency column vector and covariance matrix $\mathbf{V}$ is equivalent to the PSD matrix. When we consider the dynamics of one rigid body with 6 DOFs, the PSD matrix is given as

$$
[G(\omega)]=\frac{1}{N T} \sum_{k}^{N}\left[\begin{array}{llll}
G_{X X} & G_{X Y} & & G_{X \theta Z} \\
G_{Y X} & G_{Y Y} & & \\
& & \ddots & \\
G_{\theta Z X} & & & G_{\theta Z \theta Z}
\end{array}\right]_{k}
$$

, where $\mathrm{N}$ is the average number of FFTs, $\mathrm{T}$ is the window size of time-history, and subscripts of $\mathrm{G}$ are the direction of motion. The maximum eigenvalues corresponding to each frequency are equal to the power spectrum of the first principal component.

When the system is excited by a random input, $\varphi_{1}$ obtained from eigenvalue analysis for the PSD matrix is equivalent to the estimation of modal vector $\boldsymbol{\varphi}_{r}$. Here, the r-th SDOF 
PSD is given as follows on the assumption that the damping characteristics are represented as proportional viscous damping.

$$
G(\omega)=\frac{A_{r}}{\left\{1-\left(\frac{\omega}{\Omega_{r}}\right)^{2}\right\}^{2}+4 \zeta_{r}{ }^{2}\left(\frac{\omega}{\Omega_{r}}\right)^{2}}
$$

,where $\mathrm{Ar}$ is a positive real number corresponding to the $\mathrm{r}$-th modal vector, $\Omega \mathrm{r}$ is the undamped angular frequency, and $\zeta$ is the modal damping ratio. As a result, the modal parameters $\operatorname{Ar}, \Omega \mathrm{r}$, and $\zeta \mathrm{r}$ are identified by non-linear least-squares analysis focusing on peaks of PSD eigenvalues.

\subsection{Vibration Data Measurement}

The ISO standard ${ }^{(8)}$ for vibration measurement in elevators indicates the characteristics of a human body against the vibration, as shown in Fig. 3. In this standard, a band pass filter with a peak of around $2 \mathrm{~Hz}$ is used for evaluating ride quality. Therefore, the frequency we are interested in is under $4 \mathrm{~Hz}$.

To specify the modes during an elevator operation, we conducted experiments using a high-speed elevator with the specifications listed in Table 1. We define the $\mathrm{X}$ axis as the back and forth direction, the $\mathrm{Y}$ direction as the right and left direction, and the $\mathrm{Z}$ axis as the vertical direction. Twenty accelerometers were attached in the cabin, frame, as shown in Fig. 4. The FDD is valid for vibration produced by random input. Therefore, we set artificial disturbances on the surface of the guide rails in the $\mathrm{X}$ direction, as shown in Fig. 5. Figure 6 shows cabin and frame accelerations at the center of gravity. The accelerations at the center of gravity are calculated using Eq. (11), which translates the motion of the local position into the motion of the center of gravity in a rigid body.

$$
\left\{X_{g}\right\}=\left([R]^{T}[R]\right)^{-1}[R]^{T}\left\{X_{p}\right\}
$$

,where $\left\{X_{g}\right\}$ is the motion of the center of gravity, $[R]$ is the matrix composed of the coordinate data from the center of gravity, and $\left\{X_{p}\right\}$ is the motion of the local position.

The accelerations of the cabin and the frame are approximately the same. To clearly visualize these two motions, the accelerations and angular accelerations of the cabin and the frame are moved along the $\mathrm{X}$-axis of the base line to $\pm 0.5 \mathrm{~m} / \mathrm{s}$ and $\pm 0.5 \mathrm{rad} / \mathrm{s}$, respectively.

As a result, we treat both the cabin and the frame as one rigid body. The results from operational modal analysis are listed in Table 2 and shown in Fig. 7. In the frequencies under $4 \mathrm{~Hz}$, natural frequencies are estimated to be $1.68,2.23$, and $2.67 \mathrm{~Hz}$ representing $\mathrm{X}$-translational, Z-rotational, and Y-rotational modes, respectively. 


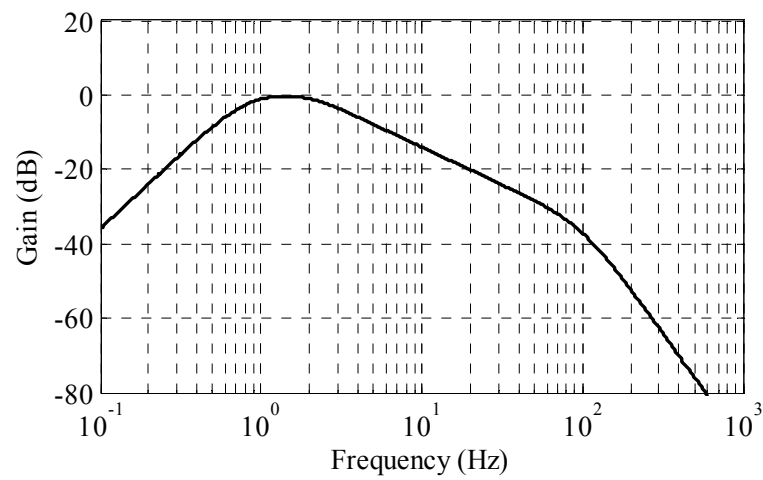

Fig. 3 Characteristics of ISO filter

Table 1 Specifications of test elevator

\begin{tabular}{|c|c|c|}
\hline \multicolumn{2}{|c|}{ Velocity } & $4 \mathrm{~m} / \mathrm{s}$ \\
\hline \multicolumn{2}{|c|}{ Stroke } & $64 \mathrm{~m}$ \\
\hline \multicolumn{2}{|c|}{ Weight } & $3074 \mathrm{~kg}$ \\
\hline \multirow{2}{*}{$\begin{array}{c}\text { Moments } \\
\text { of inertia }\end{array}$} & $\mathrm{I}_{\mathrm{xx}}$ & $8.3 \times 10^{3} \mathrm{~kg} \mathrm{~m}^{2}$ \\
\cline { 2 - 3 } & $\mathrm{I}_{\mathrm{yy}}$ & $7.7 \times 10^{3} \mathrm{~kg} \mathrm{~m}^{2}$ \\
\cline { 2 - 3 } & $\mathrm{I}_{\mathrm{zz}}$ & $2.6 \times 10^{3} \mathrm{~kg} \mathrm{~m}^{2}$ \\
\hline
\end{tabular}

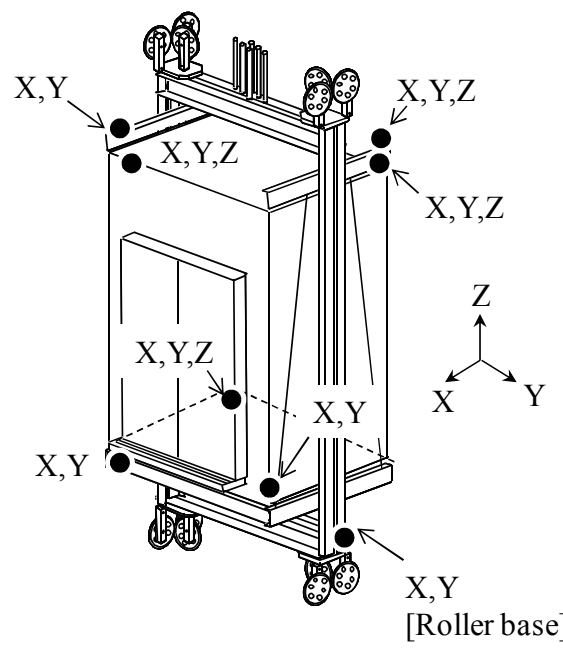

Fig. 4 Sensor arrangement

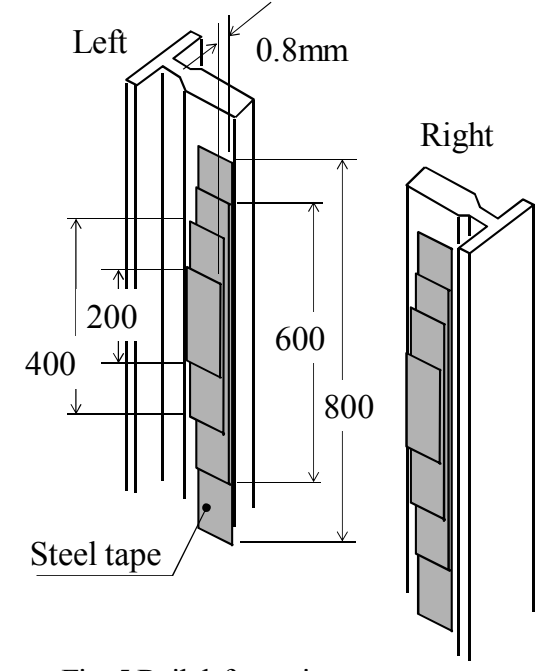

Fig. 5 Rail deformation

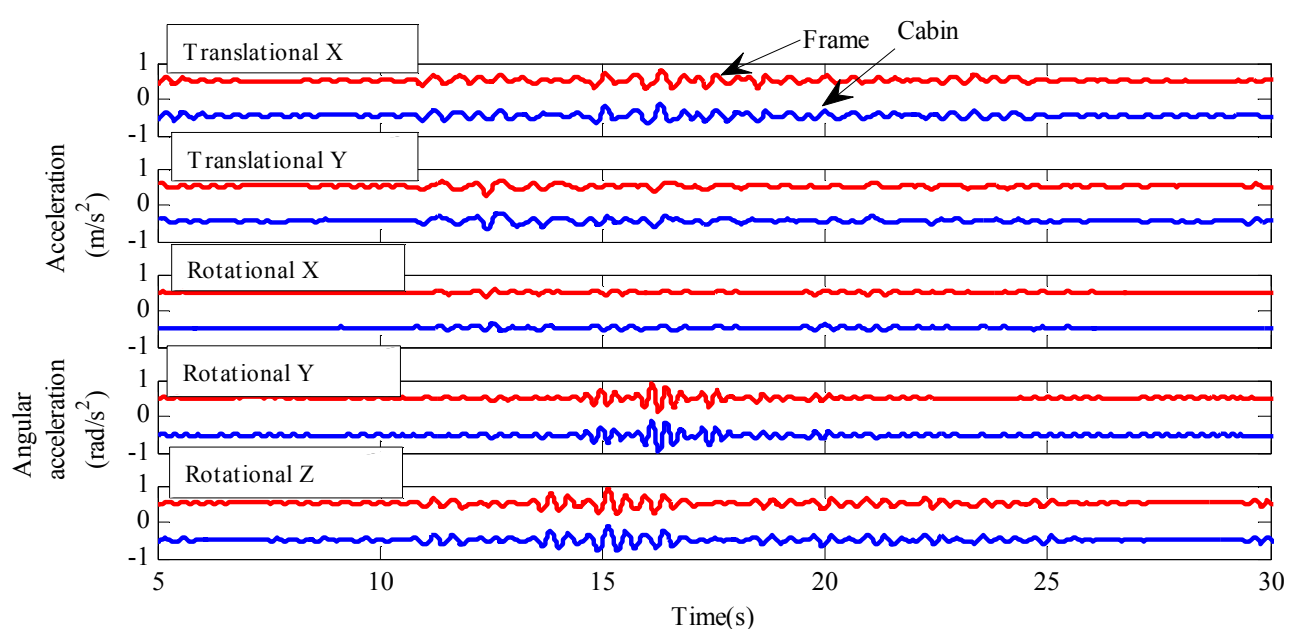

Fig. 6 Comparison of cage and frame motions 
Table 2 Identified modal parameters

\begin{tabular}{|c|c|c|c|}
\hline Order & Natural frequency $(\mathrm{Hz})$ & Damping ratio (\%) & Mode shape \\
\hline 1st & 1.68 & 3.2 & X-trans. \\
\hline 2nd & 2.23 & 3.5 & Z-rot. \\
\hline 3rd & 2.67 & 4.5 & Y-rot. \\
\hline
\end{tabular}

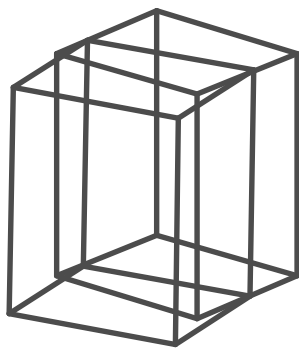

(a) $1 \mathrm{st}$

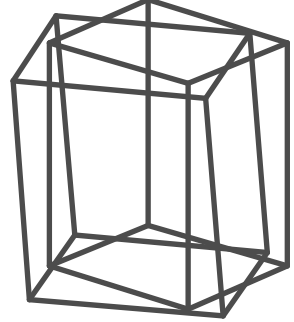

(b) 2nd

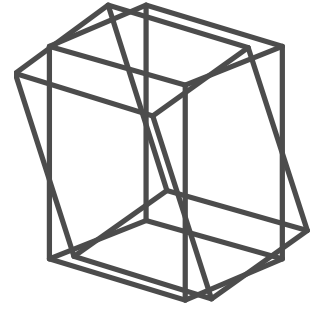

(3) $3 \mathrm{rd}$

Fig. 7 Identified mode shapes

\section{Active vibration control system}

\subsection{Mechanical System}

Roller guides are installed at four corners of a frame. One roller guide is composed of three rollers and three levers. Therefore, twelve actuators are required when each roller is controlled independently. Our proposed active roller guide system is shown in Fig. 8. The area shaded in gray is a drive incorporated in a passive roller guide without actuators. The drive is composed of an AC servomotor and ball screw. The drive presses the guide levers via springs between the lever and the saddle and produces reaction force on the car. In this system, one actuator in the $\mathrm{X}$ direction can move two rollers.

Figure 9 represents a bottom view of an elevator with our proposed active roller guide compared with the conventional system. Figure 9(a) shows the conventional active control system, which moves six rollers independently, and Fig. 9(b) shows our proposed system.

Two actuators are installed on both sides of the car in the X direction, as shown in Fig. 8. Since each roller located on both sides of the car in the $\mathrm{Y}$ direction is arranged on the same axis, one actuator is installed on one side of the car. As a result, the total number of the actuators is reduced from six to three.

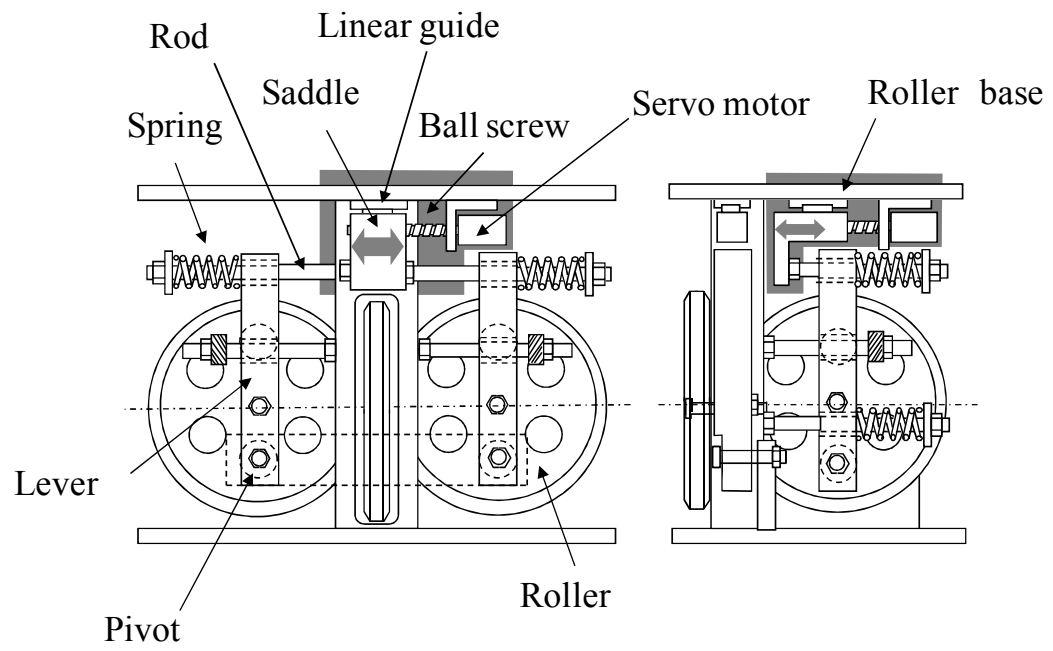

Fig. 8 Active roller guide system 


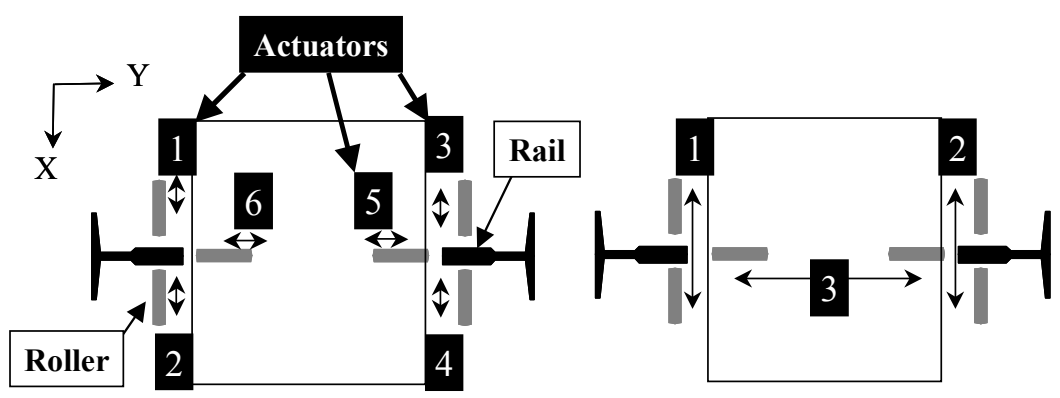

(a) Conventional system

(b) Proposed system

Fig. 9 Block diagrams of active roller guide systems

\subsection{Actuator Arrangement}

We consider whether we should arrange our proposed active control system in the upper or lower areas of the car for reducing the number of actuators. We calculated the frequency responses from the rail disturbance to the acceleration of the car using the mathematical model shown in Fig. 10. In this model, the stiffness and damping of the rollers and springs are assumed to be liner. Each of the stiffness and the damping is estimated using an optimization calculation called the Gauss-Newton method. The differences between the examination results and estimated values in the natural frequencies, and the modal assurance criterions are minimized with this method. We assume that the damping ratio is

$$
\zeta=\frac{1}{2}\left(\frac{\alpha}{\omega_{n}}+\beta \omega_{n}\right)
$$

,where $\alpha$ and $\beta$ are the coefficients of the proportional viscous damping.

Table 3 compares the stiffness values produced by optimization with the initial values. Furthermore, Table 4 compares the natural frequencies from the experiment and simulation. Two cases using the initial values and optimized values listed in Table 3 were evaluated in the simulation. The differences between the experiment and simulation were reduced from about $20 \%$ to less than $10 \%$ by using optimized values.

Figure 11 indicates the frequency responses from the disturbances caused by rail deformations to car acceleration (case 1: without damper, case 2: with damper in the upper area, case 3: with damper in the lower area). The evaluation position in the frequency responses is the floor of the car. The rail disturbances are considered as the input signal transferred by way of the guide rollers in the simulation. In Fig.11, red lines represent the responses from the lower disturbances via the lower roller guides, and blue lines represent the responses from the upper disturbances via the upper roller guides.

Since the center of gravity is in the lower area of the car, the effect of the damper in the 3 rd mode is larger than that in the 1 st and 2 nd modes. In particular, there is more suppression of acceleration in case 2 than that in case 3. Therefore, the dampers are arranged in the upper area for suppressing the 3rd mode considering cost-effectiveness.

Since the effect of the damper is small in the 1 st and 2 nd modes, we installed our proposed active control system. Figure 11 indicate that lower disturbance is more sensitive to car acceleration compared with upper disturbance. Therefore, we installed our proposed system in the lower area of the car, which has a larger effect for suppressing car acceleration. 


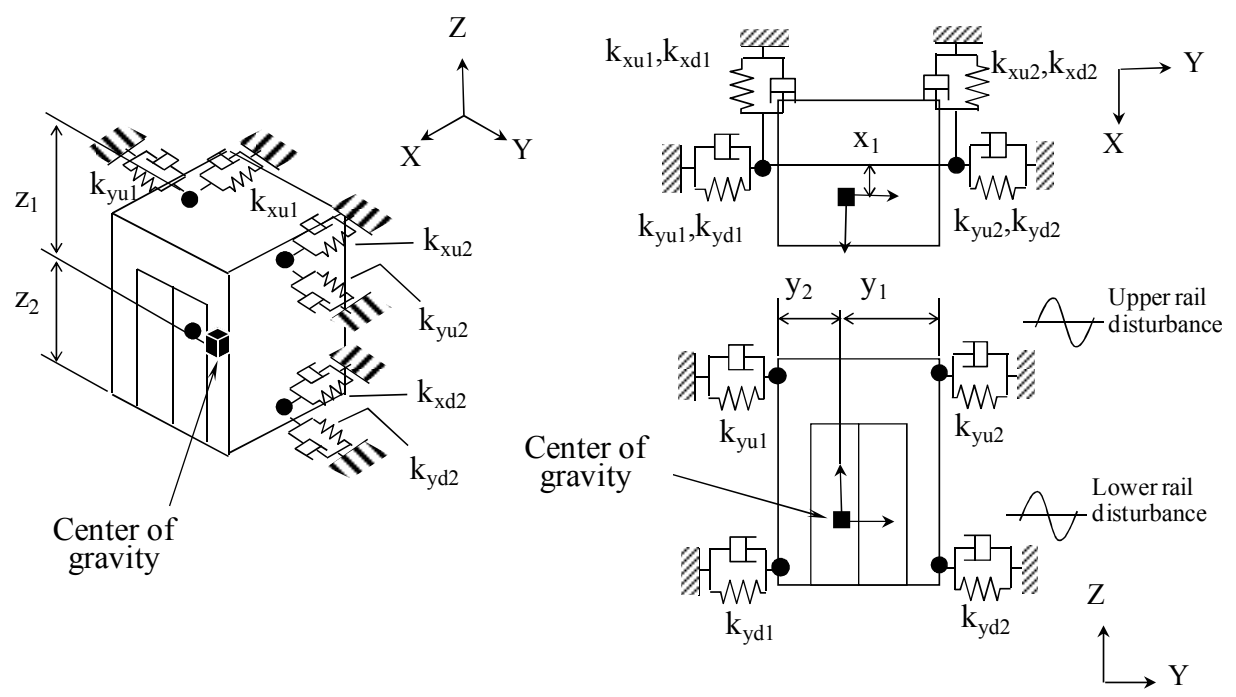

Fig. 10 Dynamic model of elevator car

Table 3 Estimation results (\%)

\begin{tabular}{|c|c|c|c|}
\hline \multicolumn{4}{|c|}{ Stiffness (proportion of initial value) } \\
\hline $\mathrm{k}_{\mathrm{xu} 1}$ & 80.0 & $\mathrm{k}_{\mathrm{xu} 2}$ & 80.0 \\
\hline $\mathrm{k}_{\mathrm{xd} 1}$ & 88.6 & $\mathrm{k}_{\mathrm{xd} 2}$ & 85.2 \\
\hline $\mathrm{k}_{\mathrm{yu} 1}$ & 111.2 & $\mathrm{k}_{\mathrm{yu} 2}$ & 111.2 \\
\hline $\mathrm{k}_{\mathrm{yd} 1}$ & 84.5 & $\mathrm{k}_{\mathrm{yd} 2}$ & 84.5 \\
\hline
\end{tabular}

Table 4 Estimation results of natural frequency $(\mathrm{Hz})$

\begin{tabular}{|c|c|c|c|}
\hline \multirow{2}{*}{ Order } & \multirow{2}{*}{ Experiment } & \multicolumn{2}{|c|}{ Model } \\
\cline { 3 - 4 } & & (a) By initial value & (b) By optimized value \\
\hline 1st & 1.58 & 1.89 & 1.74 \\
\hline 2nd & 2.13 & 2.19 & 2.00 \\
\hline 3rd & 2.66 & 3.05 & 2.77 \\
\hline
\end{tabular}

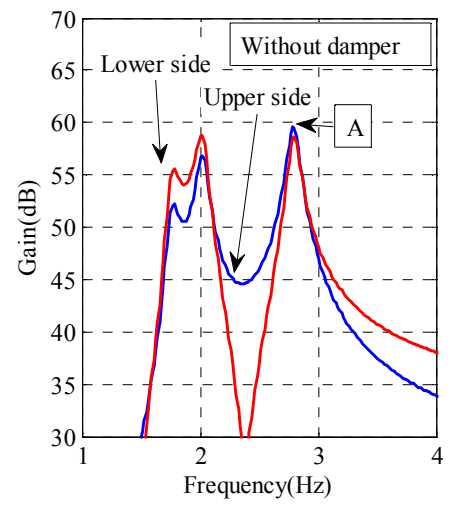

(a) case 1

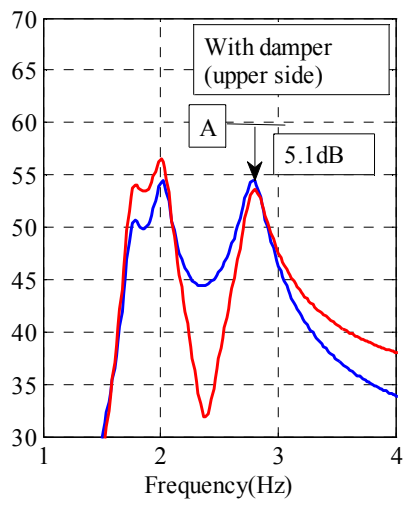

(b) case 2

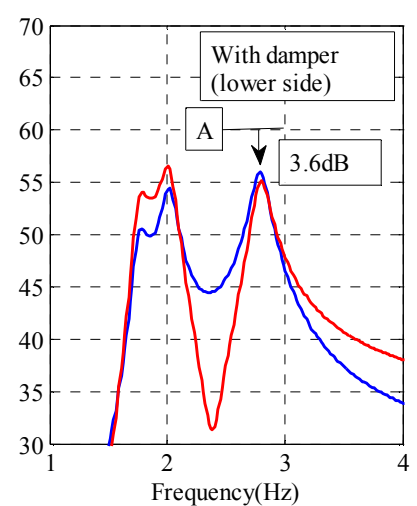

(c) case 3

Fig. 11 Transfer functions of floor acceleration by rail disturbances in passive system 


\section{Controller Design}

\subsection{Elevator model for control}

An elevator car without our proposed active control system has at least 22 DOFs, except for vertical motion. The cabin and frame each have 5 DOFs, and the four roller guides installed at the four corners each have 3 DOFs. To simplify the controller, the model order representing the elevator dynamics with 22 DOFs is reduced. The models are shown in Fig.

12. Three features are summarized below.

(1) Both the cabin and frame are treated as one rigid body

(2) The X-translational, Z-rotational, and Y-rotational modes are represented as model A

(3) The Y-translational, X-rotational modes are represented as model B

In these models, the stiffness and damping of the roller guides are assumed to be linear.

The actuators are represented as equivalent masses and springs.

The equation of motion is

$$
M_{(i)} \ddot{q}_{(i)}+C_{(i)} \dot{q}_{(i)}+K_{(i)} q_{(i)}=f_{d(i)}+f_{a(i)}
$$

,where $M_{(i)}, C_{(i)}$, and $K_{(i)}$ are mass, damping, and stiffness, respectively.

$f_{d(i)}, f_{a(i)}$ represent the rail disturbances and control force, respectively, and $i$ is the number for switching the model depending on the motion.

That is, $\mathrm{Eq}(13)$ represents model $\mathrm{A}$ when $\mathrm{i}=1$ and model $\mathrm{B}$ when $\mathrm{i}=2$.

Vector $q_{(1)}$ is

$$
q_{(1)}=\left[\begin{array}{lllllll}
x & \theta_{y} & \theta_{z} & x_{a L} & x_{a R} & \theta_{l L} & \theta_{l R}
\end{array}\right]^{t}
$$

,and vector $q_{(2)}$ is

$$
q_{(2)}=\left[\begin{array}{llll}
y & \theta_{x} & \theta_{l} & y_{a}
\end{array}\right]^{t}
$$

The major notations in these models are as follows :

$$
\begin{array}{ll}
m, I_{x}, I_{y}, I_{z} & : \text { car mass and moments of inertia } \\
m_{a} & : \text { equivalent mass of actuator } \\
k_{u}, k_{L}, k_{R} & : \text { spring constants of upper roller guides } \\
k_{d l} & : \text { spring constant of lower roller guide(left side) } \\
k_{r R}, k_{r L}, k_{r} & : \text { spring constants of lower rollers } \\
k_{d R}, k_{d L}, k_{l}, k_{d} & : \text { spring constants of lower guide springs } \\
k_{a R}, k_{a L}, k_{a} & : \text { spring constants of actuators }
\end{array}
$$

The total degrees of freedom in these models are 11, 7 in model A and 4 in model B. 
<smiles>[Y]C([Y])C([Y])[Y]</smiles>

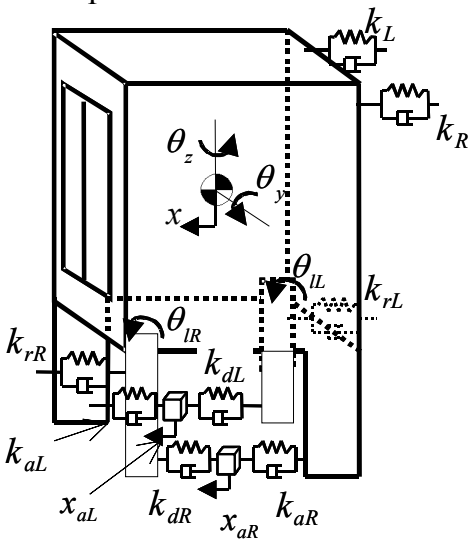

(a) Model A

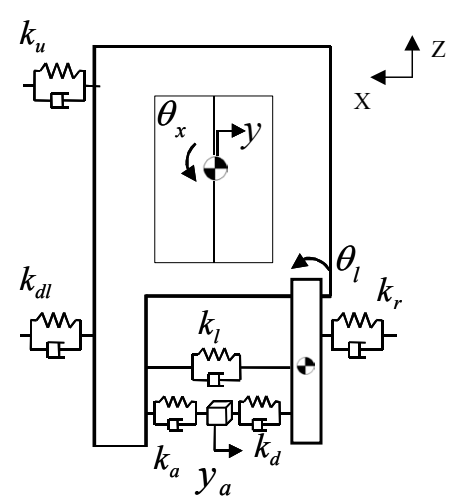

(b) Model B

Fig. 12 Dynamic models for control

\subsection{Estimation of parameters}

The stiffness and delay of the actuator with the ball screw are included in the models. The actuator model is composed of an equivalent mass and a spring, and including the 4th order transfer function representing the time delay of the actuator. A shaking test by actuators was conducted to estimate the dynamic parameters. The relative displacement between the saddle and frame was measured with a laser displacement meter, as shown in Fig. 13. The acceleration shown in Fig. 2 was also measured at the same time. The FRFs between the acceleration and relative displacement were calculated in elevator operation.

The masses and moments of inertia are given. The spring constants, such as $k_{L}$ and $k_{R}$, tend to change according to the contact condition between the guide rails and guide rollers. Therefore, these values are estimated by minimizing the differences between the experiment and simulation in the gains of FRFs. Figure 14 shows FRFs by the experiment and simulation using model A. Table 5 compares the values of the stiffness obtained by optimization compared with the initial values. Furthermore, Table 6 compares the natural frequencies between the experiment and simulation. Two cases using the initial values and optimized values listed in Table 5 were evaluated in the simulation. The results indicate that FRFs and natural frequencies calculated by using the optimized values are approximately same as those of the experiment.

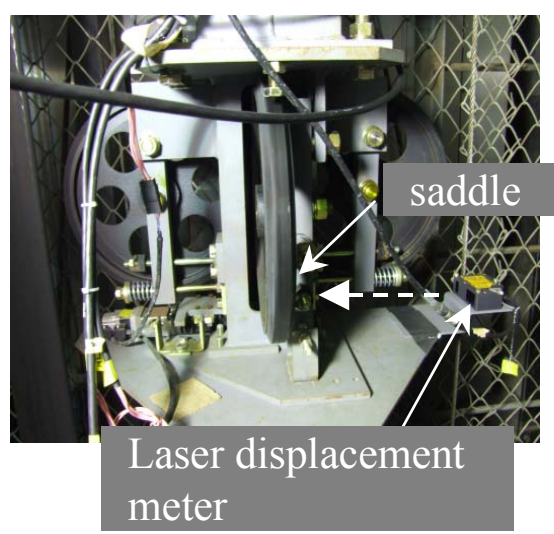

Fig. 13 Measurement of displacement 

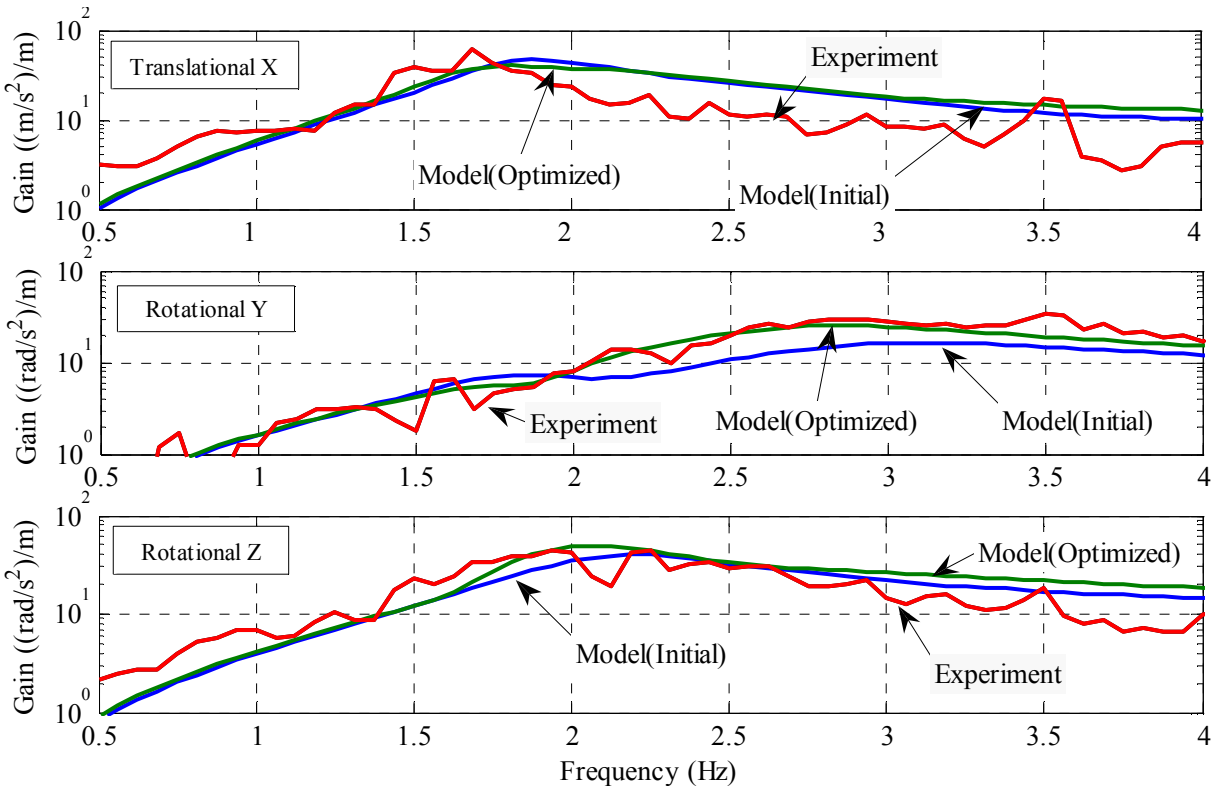

Fig. 14 Comparison of experiment and simulation

Table 5 Estimation results

\begin{tabular}{|c|c|c|c|}
\hline No. & \multicolumn{2}{|c|}{ Variables } & Value(\%) \\
\hline \multirow{4}{*}{1} & & $k_{L}$ & 80 \\
& Stiffness & $k_{R}$ & 80 \\
& (proportion of initial value) & $k_{d L}$ & 97 \\
& & $k_{d R}$ & 80 \\
\hline \multirow{2}{*}{2} & Damping & $\beta$ & 0.022 \\
\hline
\end{tabular}

Table 6 Estimation results of natural frequency $(\mathrm{Hz})$

\begin{tabular}{|c|c|c|c|}
\hline \multirow{2}{*}{ Order } & \multirow{2}{*}{ Experiment } & \multicolumn{2}{|c|}{ Model } \\
\cline { 3 - 4 } & & (a) By initial value & (b) By optimized value \\
\hline 1 & 1.78 & 1.85 & 1.76 \\
\hline 2 & 1.95 & 2.15 & 2.05 \\
\hline 3 & 2.82 & 2.99 & 2.78 \\
\hline
\end{tabular}

\subsection{Controller design}

Our proposed active control system is shown in Fig. 15. The three actuators are installed at the lower area of the car, and two biaxial accelerometers are attached to the frame and roller base. Assuming the full controller model, the numbers of the controller is total 12 multiplying 3(the numbers of actuators) by 4(the numbers of measurement value). In this system, we divide the controller into two types by using the characteristics of decoupling in vibrational modes. One is a controller for suppressing X-translational, Y-rotational, and Z-rotational modes. The other is a controller for suppressing Y-translational and $\mathrm{X}$-rotational modes. As a result, number of controllers is reduced from 12 to $5(=4+1)$.

Since the natural frequency of the 1st mode is changed from 1.6 to $1.3 \mathrm{~Hz}$ according to the car load, the controller must be designed in consideration of system stability due to this change. Moreover, a person will likely feel low frequency vibration. An H-infinity control design method is used to maintain the stability of the system when dealing with a change in 
load and achieve robust performance under $4 \mathrm{~Hz}$. The design of the controller is based on the block diagram shown in Fig. 16. $\mathbf{P}$ represents the plant model of the elevator. A feedback controller $\mathbf{K}$ is determined to minimize the $\mathrm{H}$-infinity norm from disturbances $\boldsymbol{\omega}$ to $\mathbf{z}$. $\boldsymbol{\omega}$ is composed of the rail disturbances $\boldsymbol{\omega}_{r}$, input noise $\mathbf{p}$, and sensor noise $\mathbf{n} . \mathbf{z}$ is composed of the car acceleration $\mathbf{z}_{1}$ and the control output $\mathbf{z}_{2}$. Three weighting functions used in the controller design are shown in Fig. 17. Wp is a band pass filter representing the sensitivity of the human body. Wr is a band pass filter for achieving robust stability. Furthermore, a filter $\mathbf{F}$ is introduced to eliminate sensor noise. By using these characteristics, the controller is designed to minimize the $\mathrm{H}$-infinity norm. Figure 18 represents four H-infinity controllers from the acceleration of each sensor to each actuator with the 46-th order of the controller. To simplify the controller, we determine the proportional gain of the $\mathrm{P}$ controller by averaging the $\mathrm{H}$-infinity controller gain from 1 to $4 \mathrm{~Hz}$. Figure 19 represents the closed-loop characteristics from the rail disturbances to car accerelation. These conditions are $0 \%$ load and $100 \%$ load within the frequency area including the 1 st and 2 nd modes. This simplified controller suppresses vibration.
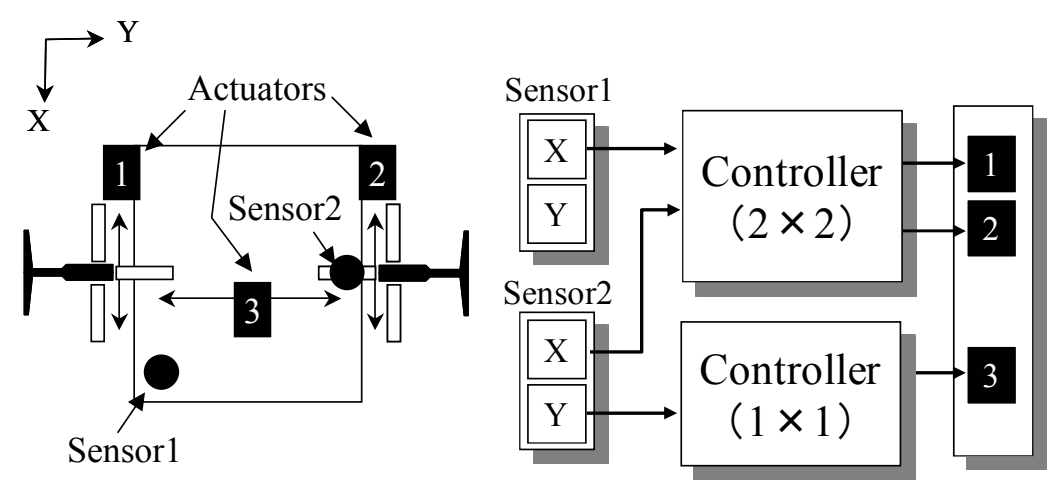

Fig. 15 Block diagrams of proposed active control system

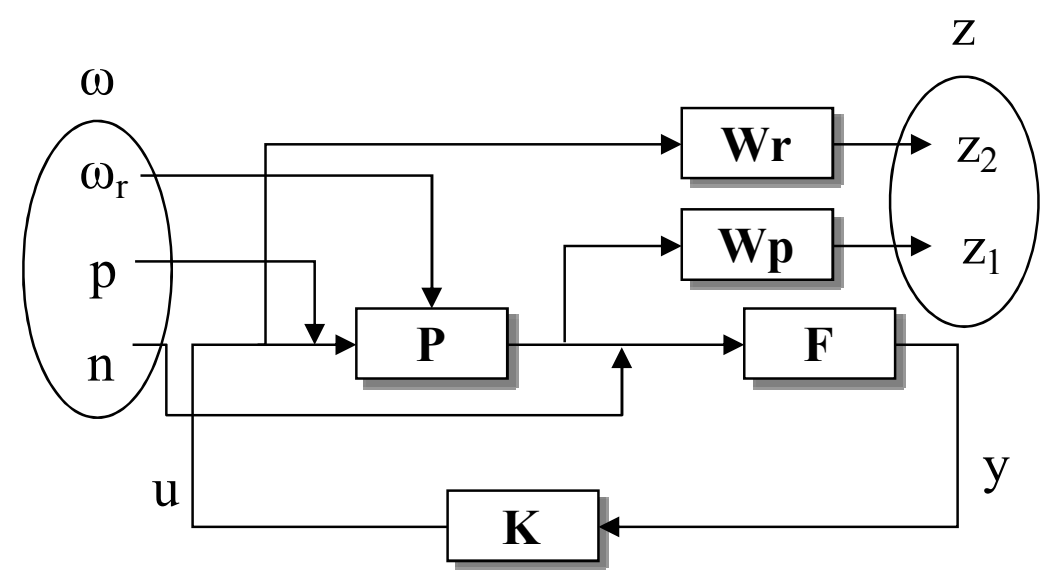

Fig. 16 Block diagram of H-infinity controller 

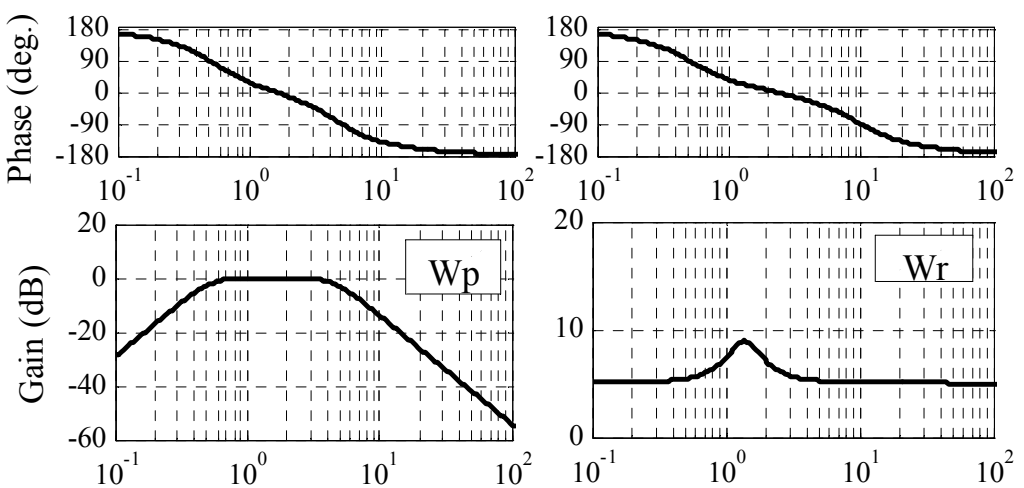

Frequency $(\mathrm{Hz})$

Frequency $(\mathrm{Hz})$
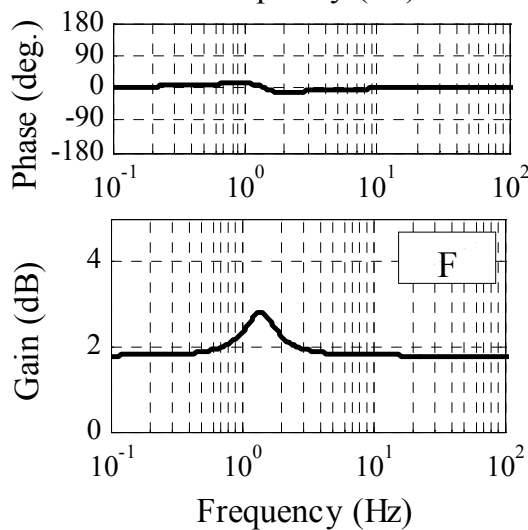

Fig. 17 Weighting function
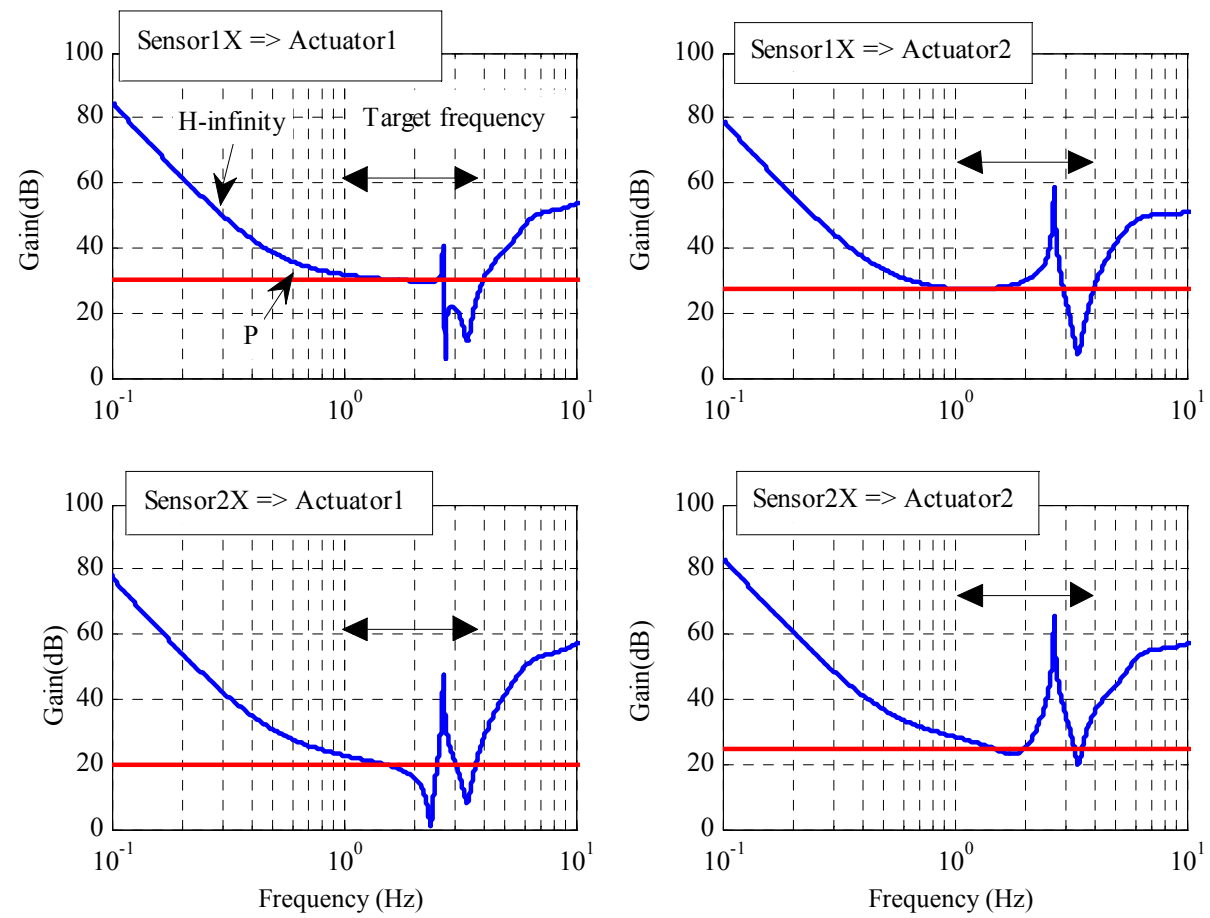

Fig. 18 Controller gains of $\mathrm{H} \infty$ controller and $\mathrm{P}$ controller 

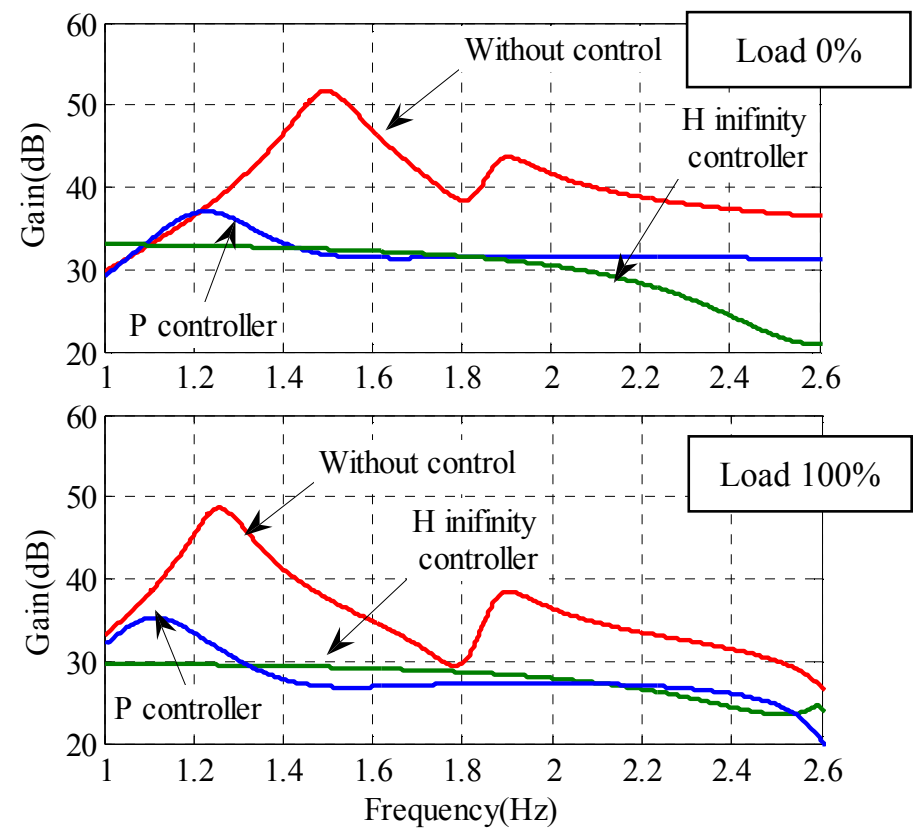

Fig. 19 Closed-loop transfer function from rail disturbance to floor acceleration in simulation

\section{Experiment}

To evaluate the performance of our proposed active control system, we carried out on-site experiments. The elevator car speed was $4 \mathrm{~m} / \mathrm{s}$. Figure 20 shows the vibrations "with control" and "without control". These results show that our proposed active control system can reduce vibrations by almost half the amplitude of the vibrations of the cabin. 

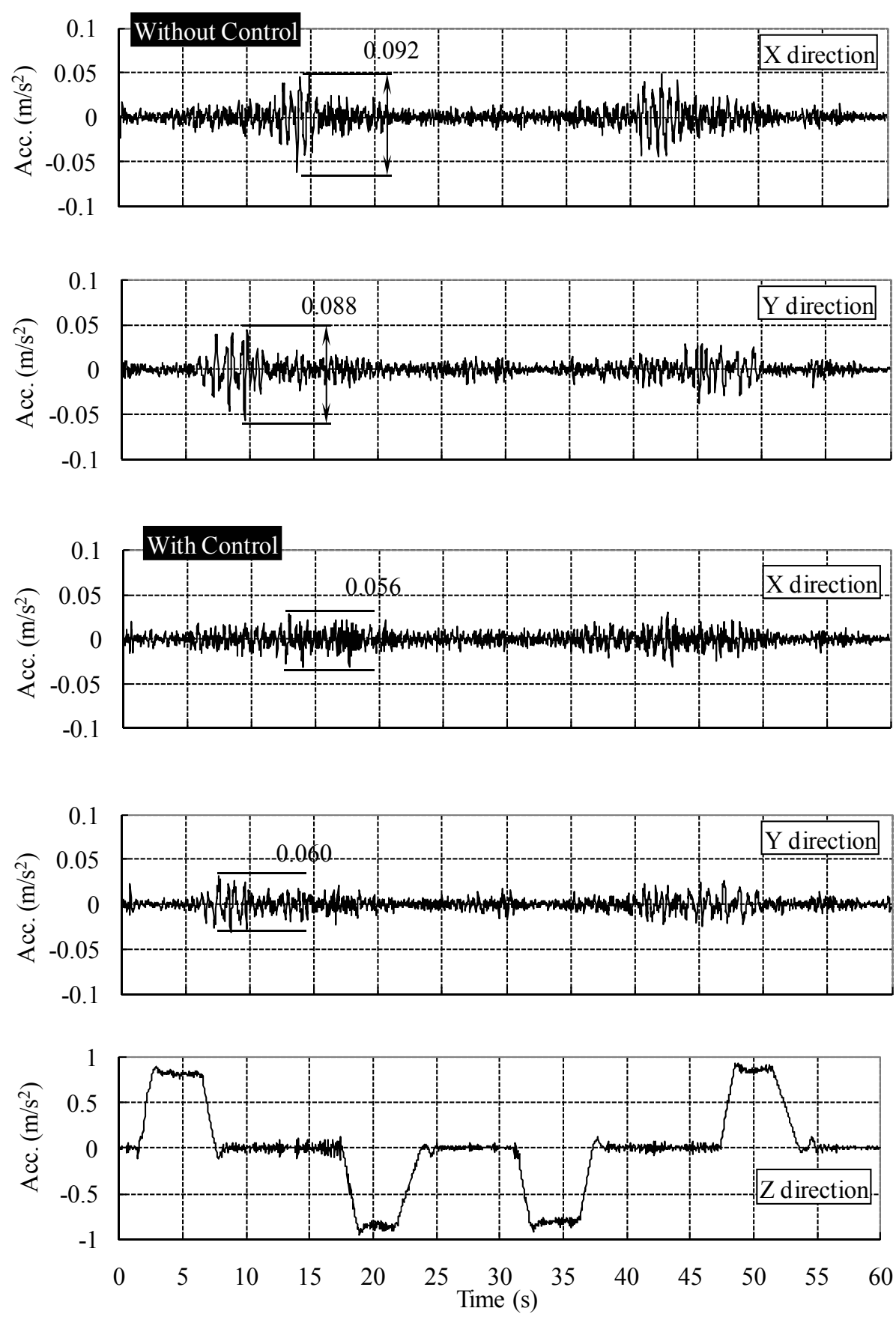

Fig. 20 Experimental results

\section{Conclusion}

We developed an active control system for high-speed elevators to improve ride quality. We obtained the following results:

(1) Primary vibrational modes were identified under $4 \mathrm{~Hz}$ from operational modal analysis.

(2) A new mechanical system composed of three actuators was developed.

(3) Low dimensional models of 11 DOFs were introduced for the controller design.

(4) P controller gains were determined by averaging the $\mathrm{H}$-infinity controller gains under 4 Hz to simplfy the controller.

(5) Our proposed active control system suppressed vibrations by almost half the amplitude of vibrations of the cabin. 


\section{Acknowledgements}

We would like to thank Mr. Shigeyuki Kobayashi who is a member of dynamics laboratory at Tokyo metropolitan university under the leadership of Prof. T. Yoshimura. His work on analysis of vibration data is gratefully acknowledged.

\section{References}

(1) Mutoh N, Kagomiya K, Kurosawa T, Konya M, Andoh T, Horizontal Vibration Suppression Method Suitable for Super-High-Speed Elevators, Transactions of the Institute of Electrical Engineers of Japan, Series D, Vol. 118, No.3 (1998), pp. 353-362

(2) Toyoshima N, Kamimura K, Hirai M, Nagai M, Kamata T, Vibration Control of Ultra High Speed Elevator by Active Mass Damper, Transactions of the Japan Society of Mechanical Engineers, Series C, Vol. 66, No.647 (2000), pp. 2181-2186.

(3) Utsunomiya K, Okamoto K, Yumura T, Sakuma Y, Vibration Control of High-speed Elevators Taking Account of Electricity Consumption Reduction, Transactions of the Japan Society of Mechanical Engineers, Series C, Vol. 72, No.719 (2006), pp. 2048-2055.

(4) Yamazaki Y, Tomisawa M, Okada K, Sugiyama M, Kojima K, Saragai K, Vibration Control of Super-High-Speed Elevators, Transactions of the Japan Society of Mechanical Engineers, Series C, Vol. 61, No.581 (1995), pp. 15-21.

(5) Rune Brincker, Lingmi Zhang, Palle Andersen, Modal Identification from Ambient Responses using Frequency Domain Decomposition, Proceedings of IMAC-XV III: A Conference on Structural Dynamics, Vol.1(2000-2), pp.625-629.

(6) Nis Meller, Rune Brincker, Palle Andersen, Modal Extraction on a Diesel Engine In Operation, Proceedings of IMAC-XV III : A Conference on Structural Dynamics, Vol.1(2000-2), pp.1845-1851.

(7) Kobayashi S, Takuya Y, Naoaki N, Akihiro O, Estimation of dynamic characteristics of an elevator car using operational modal analysis, Transactions of the Japan Society of Mechanical Engineers, Series C, Vol. 74, No.739 (2008), pp. 548-553.

(8) ISO18738 INTERNATIONAL STANDARD LIFTS(elevators)-Measurement of Lift ride quality 